\title{
BMJ Open Transmission dynamics and the effects of non-pharmaceutical interventions in the COVID-19 outbreak resurged in Beijing, China: a descriptive and modelling study
}

Xiaoming Cui, ${ }^{1}$ Lin Zhao (1) , ${ }^{2}$ Yuhao Zhou (1) , ${ }^{1}$ Xin Lin,,${ }^{3,4}$ Runze Ye, ${ }^{2} \mathrm{Ke} \mathrm{Ma},{ }^{1}$ Jia-Fu Jiang, ${ }^{1}$ Baogui Jiang, ${ }^{1}$ Zhang Xiong, ${ }^{5}$ HongHao Shi, ${ }^{3}$ Jingyuan Wang, ${ }^{3,4}$ $\mathrm{Na} \mathrm{Jia},{ }^{1}$ Wuchun $\mathrm{Cao}^{1,2}$

To cite: Cui X, Zhao L, Zhou Y, et al. Transmission dynamics and the effects of nonpharmaceutical interventions in the COVID-19 outbreak resurged in Beijing, China: a descriptive and modelling study. BMJ Open 2021;11:e047227. doi:10.1136/ bmjopen-2020-047227

- Prepublication history and additional supplemental material for this paper are available online. To view these files, please visit the journal online. (http://dx.doi.org/10.1136/ bmjopen-2020-047227).

$\mathrm{XC}, \mathrm{LZ}, \mathrm{YZ}$ and $\mathrm{XL}$ contributed equally.

Received 24 November 2020 Accepted 28 July 2021

Check for updates

(c) Author(s) (or their employer(s)) 2021. Re-use permitted under CC BY-NC. No commercial re-use. See rights and permissions. Published by BMJ.

For numbered affiliations see end of article.

Correspondence to

Dr Jingyuan Wang;

jywang@buaa.edu.cn,

Dr Na Jia;

jiana79_41@hotmail.com and

Dr Wuchun Cao

caowuchun@126.com

\section{ABSTRACT}

Objective To evaluate epidemiological characteristics and transmission dynamics of COVID-19 outbreak resurged in Beijing and to assess the effects of three nonpharmaceutical interventions.

Design Descriptive and modelling study based on surveillance data of COVID-19 in Beijing.

Setting Outbreak in Beijing.

Participants The database included 335 confirmed cases of COVID-19.

Methods To conduct spatiotemporal analyses of the outbreak, we collected individual records on laboratoryconfirmed cases of COVID-19 from 11 June 2020 to 5 July 2020 in Beijing, and visitor flow and products transportation data of Xinfadi Wholesale Market. We also built a modified susceptible-exposed-infected-removed model to investigate the effect of interventions deployed in Beijing.

Results We found that the staff working in the market $(52.2 \%)$ and the people around $10 \mathrm{~km}$ to this epicentre $(72.5 \%)$ were most affected, and the population mobility entering-exiting Xinfadi Wholesale Market significantly contributed to the spread of COVID-19 $(p=0.021)$, but goods flow of the market had little impact on the virus spread $(p=0.184)$. The prompt identification of Xinfadi Wholesale Market as the infection source could have avoided a total of 25708 (95\% Cl 13657 to 40 625) cases if unnoticed transmission lasted for a month. Based on the model, we found that active screening on targeted population by nucleic acid testing alone had the most significant effect.

Conclusions The non-pharmaceutical interventions deployed in Beijing, including localised lockdown, closecontact tracing and community-based testing, were proved to be effective enough to contain the outbreak. Beijing has achieved an optimal balance between epidemic containment and economic protection.

\section{INTRODUCTION}

The COVID-19 pandemic, caused by SARS-CoV-2, is the most significant public

\section{Strengths and limitations of this study}

- We established the database including 335 confirmed cases of COVID-19 with detailed information.

- We comprehensively assessed epidemiological and spatiotemporal characteristics of COVID-19 outbreak in Xinfadi Wholesale Market.

- We developed a modified epidemiological model with time-variant parameters inferred from data for in-depth analysis on the effects of nonpharmaceutical interventions.

- Our model did not contain the spatial transmission of the epidemic due to the lack of inter-regional migration data.

- Our model only compared the most important interventions out of tens of control measures implemented in Beijing.

health crisis in a century and has infected $>135$ million people with nearly 3 million dead worldwide. ${ }^{1}$ SARS-CoV-2 was initially thought to be linked to Huanan Seafood Wholesale Market in Wuhan, China, ${ }^{2}$ although multiple origins later suggested for the human emergence. ${ }^{34}$ Recently, another Chinese market, Xinfadi Wholesale Market, has been in the hot topic. The market is linked to a surge in COVID-19 cases in Beijing after 56-day stretch without any local transmitted cases, ${ }^{5}$ triggering fears of a second wave of COVID-19 in mainland China. WHO called the new cluster in Beijing 'a significant event' that needs to be investigated and controlled. ${ }^{6}$

This event has further raised a critical question, why the wholesale market is frequently associated with the emergence of a human infection outbreak, as observed in SARS-CoV-2, SARS-CoV ${ }^{7}$ and avian influenza A (H7N9). ${ }^{8}$ Identifying factors that promote 
maintenance, amplification and dissemination of virus occurred in the wholesale market has significant public health consequences. Additionally, it is sophisticated to develop control strategies to curtail the transmission of COVID-19, which fit the epidemiological situations, capacities and values of each country. The wholesale market characterises as the complex and high density of stalls, relatively poor environmental sanitation and daily crowded human activities. The virus might be quickly transmitted either by extensive exposed human or contaminated goods. How to promptly contain the disease spread, return to a normal life and keep the local economy alive is a difficult trade-off problem.

In this study, we aimed to describe the epidemiological characteristics of COVID-19 outbreak linked to Xinfadi Wholesale Market in Beijing, and to analyse the key factors that contributed to prompt local transmission. We also built a modified transmission model, which is more in line with the real dynamic epidemic prevention and control, to evaluate the effect of non-pharmaceutical interventions (NPIs) implemented in Beijing, including localised lockdown, close-contact tracing and communitybased nucleic acid testing (NAT).

\section{METHODS}

\section{Data collection}

Individual records on COVID-19 cases were collected from official reports by Beijing Center for Disease Prevention and Control (https://www.bjcdc.org) from 11 June 2020 to 5 July 2020. Demographic characteristics (age, sex, occupation and residence location), exposure history, identification approach, clinical classification and key time points, including date of exposure to Xinfadi Wholesale Market, symptom onset, hospital admission and reporting were extracted. Data on visitor and goods flow volume from Xinfadi Wholesale Market to each district were obtained from Aurora Big Data (Shenzhen Hexun Huagu Information Technology) and Liu's thesis (online supplemental table S1, online supplemental table S2). ${ }^{9}$ Aggregated and anonymised traffic volumes of all subdistricts in Beijing were collected from an online ridehailing platform. Data of NPIs were obtained from the National Health Commission of the People's Republic of China (http://en.nhc.gov.cn) and Beijing Municipal Health Commission (http://wjw.beijing.gov.cn/).

\section{Spatiotemporal analyses}

Thematic maps showing the distribution of confirmed cases in Beijing, and visitor and goods flow from Xinfadi Wholesale Market to each district or subdistrict were produced by ArcGIS V.10.2 software (ESRI, Redlands, California, USA). We measured the commuting distance between each COVID-19 case and Xinfadi Wholesale Market based on city's transportation network. Particular locations of residential communities of 292 confirmed cases were extracted for mapping, and administrative agency of subdistricts where other 43 cases lived were used when exact locations were not available. The distancedate-case matrix was used to illustrate the relationships between the number of confirmed cases and commuting distances from Xinfadi Wholesale Market and the date of symptom onset.

\section{Estimation of effective reproduction number}

We estimated the effective or net reproduction number on day $\mathrm{t}\left(R_{t}\right)$, which is defined as the mean number of secondary cases generated by one primary case with illness onset on day t using the method developed by Cori et al (online supplemental text 1 ) ${ }^{10}$ CIs of $R_{t}$ were quantified using a bootstrap procedure. ${ }^{11}$

\section{Simulation model}

We adopt a modified susceptible-exposed-infectedremoved (SEIR) model to simulate the effects of different NPIs (online supplemental figure S1). This model divides the population into six compartments, that is, the susceptible population $S$, the exposed population $E$, the presymptomatic population $P$, the symptomatic but unconfirmed population $I$, the symptomatic but previously isolated population $I S$ and the removed population $R$, which contains individuals removed from the disease transmission due to death/recovery and includes individuals that are isolated once they obtain laboratory confirmation. The extension on the definition of compartment $R$ is based on the actual situation in China, where once the infected persons obtain laboratory confirmation, they will be quarantined and would not cause secondary infection anymore. The dynamics of these compartments across time $t$ could be summarised by the following set of ordinary differential equations in Eq. (1):

$$
\begin{aligned}
& \frac{d S_{t}}{d t}=-\beta_{t} \frac{S_{t}}{N} I_{t}-q \beta_{t} \frac{S_{t}}{N} P_{t} \\
& \frac{d E_{t}}{d t}=\beta_{t} \frac{S_{t}}{N} I_{t}+q \beta_{t} \frac{S_{t}}{N} P_{t}-\gamma_{e} E_{t} \\
& \frac{d P_{t}}{d t}=\gamma_{e} E_{t}-\gamma_{p} P_{t} \\
& \frac{d I_{t}}{d t}=\left(1-\alpha_{t}^{1}-\alpha_{t}^{2}\right) \gamma_{p} P_{t}-\sigma_{t} I_{t} \\
& \frac{d I S_{t}}{d t}=\left(\alpha_{t}^{1}+\alpha_{t}^{2}\right) \gamma_{p} P_{t}-\sigma_{t} I S_{t} \\
& \frac{d R_{t}}{d t}=\sigma_{t} I_{t}+\sigma_{t} I S_{t}
\end{aligned}
$$

Where $\beta_{t}$ is the transmission rate weighted by the timevariant mobility intensity $m_{t} ; q$ is the relative ratio for transmissibility of the presymptomatic infections than the symptomatic infections; $\gamma_{e}$ is the inverse of duration from exposed to presymptomatic; $\gamma_{p}$ is the inverse of duration from presymptomatic to symptomatic, that is, the presymptomatic infectious period; $\alpha^{1}{ }_{t}$ and $\alpha^{2}$, are the proportions of the new symptomatic infections discovered through close-contact tracing and community-based NAT, respectively; $\sigma_{t}$ is the inverse of duration from symptom onset to be removed. The details of parameter calculation could be found in online supplemental text 2 and online supplemental table S3. We calibrated our model by the Metropolis-Hastings Markov chain Monte Carlo (MCMC) algorithm and assessed the convergence by the trace plot and the Gelman-Rubin diagnostic (online supplemental 
text 2). ${ }^{12} 13$ The initial settings of model parameters are summarised in online supplemental table S4.

\section{Scenario settings}

Three dynamic parameters, that is, $m, \alpha^{1}$, and $\alpha^{2}$, were introduced in our model to assess the effects of three NPIs, that is, localised lockdown, close-contact tracing and community-based NAT, respectively. We designed three types of scenarios, involving (1) none NPIs implemented, (2) only one of the NPIs implemented and (3) only one NPI not implemented (ie, implementing two of the NPIs), to evaluate the effectiveness of these NPIs in containing the epidemic. The scenario simulation was based on the assumptions that: (1) the public health resources, including the resources for isolation, closecontact tracing and the ability to diagnose infected individuals, are enough even when the cases surged; and (2) the effects of NPIs would not be impacted by the start/ stop of other NPIs. For all scenarios, we adjusted the dynamic parameters concerning the settings. We repeated the simulations based on the parameter values estimated by the 10 million MCMC iterations (online supplemental text 2) to construct the 95\% CI of the epidemic curve. The simulation results were presented as mean values and $95 \%$ CI for both daily and cumulative incidence. All analyses were based on Python software (V.3.6.0) and the PyMC package (V.2.3.8). ${ }^{14}$

\section{Sensitivity analyses}

We designed six sensitivity analyses to test the robustness of our simulation results. For each of the sensitivity analyses, we fixed parameters and initial states to be the same as the main analysis except for those mentioned below. (S1) Assume the initial number of exposed individuals $\mathrm{E}(0)=35$, which is $10 \%$ less than the observed value. (S2) Assume the initial number of exposed individuals $\mathrm{E}(0)=43$, which is $10 \%$ more than the observed value. (S3) Assume the initialisation of transmission rate $\beta_{0}=0.45$, which is $50 \%$ of the value in the main analysis. (S4) Assume the initialisation of transmission rate $\beta=1.35$, which is $150 \%$ of the value in the main analysis. (S5) Assume the incubation period fixed to 4.1 days, which is the lower $95 \%$ CI from reference. ${ }^{3}$ Correspondingly, the presymptomatic infectious period is fixed to 1.1 days, according to the previous study. ${ }^{15}{ }^{16}$ (S6) Assume the incubation period fixed to 7.0 days, which is the upper 95\% CI from reference. ${ }^{3}$ Correspondingly, the presymptomatic infectious period is fixed to 3.0 days, according to the previous study. ${ }^{15} 16$ We compared the simulation results under those models with the main analysis.

\section{Statistical analyses}

Attack rate (AR) with 95\% CI was computed using the population estimate of the National Statistical Yearbook 2019 (National Bureau of Statistics of China), and presented as number of cases per million persons. Skewed data were expressed as median (IQR or range). The categorical variables were reported as frequency $(n)$ and proportion $(\%)$. To estimate the differences between groups, Student's t-test or Wilcoxon rank-sum test was used for a continuous variable where appropriate, and the $\chi^{2}$ test was used for a categorical variable. Spearman's correlation analysis was used to measure the correlations between number of cases at different districts and commuting distance, goods flow volume and visitor flow volume. Root mean square error was used as a standard to measure the prediction results of model. All the analyses were conducted using R software, V.3.6.1, and Python software, V.3.6.0. A two-tailed $p$ value $<0.05$ was considered to be statistically significant.

\section{Patient and public involvement}

Patients and the public were not involved in the design or conduct of the study.

\section{RESULTS}

As of 5 July 2020, a total of 335 laboratory confirmed cases had been reported in Beijing, of which $55.8 \%$ were males (table 1), significantly higher than proportion of males in Wuhan $(47.7 \%, \mathrm{p}=0.003)$. During this outbreak, SARS-CoV-2 was more likely to infect young adults, with median age of 43 years (IQR 31-52), while the virus tended to infect older people in Wuhan (median age: 53, IQR 40-64) and Beijing (median age: 45, IQR 34-60) before March 2020 (online supplemental figure S2A and S2B). Over half of the cases in this outbreak were people working in Xinfadi Wholesale Market, and there were $20(6.0 \%)$ purchasing agents and $59(17.6 \%)$ customers infected in the market. About a quarter of cases were close contacts to infected people or contaminated goods or environment. We estimated a median incubation period of 5 days (range 0-16) (online supplemental text 3 , online supplemental figure S3). The median durations from symptom onset to hospital admission and reporting were 1 day (range $-10-17$ ) and 2 days (range $0-18$ ), respectively. Most identified cases $(97.9 \%)$ were mild to moderate, and no one died.

The recent outbreak has affected 47 (14.2\%) subdistricts in 11 districts (figure 1A), with an AR of 24.3 (IQR 13.9-57.8) per million persons (figure 1B). All cases were epidemiologically linked to Xinfadi Wholesale Market, which has a high daily population activity density before suspension (13 June), especially in fresh meat and beef and mutton markets (figure 1C, online supplemental figure S4). The outbreak started on 4 June. The majority of cases were exposed to Xinfadi Wholesale Market between 7 June and 11 June, and experienced symptoms about 5 days later. The index case was reported on 11 June, and the epidemic peaked around 13 June (figure 1D). After the NPIs conducted, the growth of new cases slowed down and the epidemic was brought under control. The bottom part of the distance-date-case matrix, corresponding to people living close to Xinfadi Wholesale Market, was where the largest number of cases had 


\begin{tabular}{|c|c|}
\hline Characteristics & COVID-19 cases in Beijing \\
\hline Date of report & 11 June 2020-5 July 2020 \\
\hline Number of cases & 335 \\
\hline Male (\%) & $187(55.8)$ \\
\hline Age: median (IQR) & $43(31-52)$ \\
\hline \multicolumn{2}{|l|}{ Type of cases* } \\
\hline $\begin{array}{l}\text { Working population/ } \\
\text { vendors (\%) }\end{array}$ & $175(52.2)$ \\
\hline Purchasing agents (\%) & $20(6.0)$ \\
\hline Customers (\%) & $59(17.6)$ \\
\hline Close contacts (\%) & $81(24.2)$ \\
\hline
\end{tabular}

Incubation period: median 5 (0-16)

(range)

Days from symptom onset $1(-10-17)$
to hospital admission:
median (range)

Days from symptom onset $2(0-18)$

to reporting: median

(range)

$\begin{array}{ll}\text { Clinical classification } & \\ \text { Mild } & 57(17.0 \%) \\ \text { Moderate } & 271(80.9 \%) \\ \text { Severe } & 4(1.2 \%) \\ \text { Critical } & 3(0.9 \%)\end{array}$

${ }^{*}$ Classified by the exposure history to Xinfadi Wholesale Market. Working population/Vendors, people working in the market; purchasing agents, people purchasing at the market for smaller wholesale markets, restaurants, supermarket chains, stalls and online shopping platforms; customers, people buying at the market for individuals; close contacts, people contacting with infected persons or contaminated goods from Xinfadi Wholesale Market.

been recorded (figure 1E), especially within $10 \mathrm{~km}$ from the market $(72.5 \%$ ) (online supplemental figure S5A-C).

Figure 2A indicates that no significant correlation was found between number of cases and goods flow volume $(\mathrm{p}=0.184)$. The agricultural products from Xinfadi Wholesale Market are mainly supplied to the northern districts, including Chaoyang, Haidian Districts and the surrounding Fengtai, Xicheng and Dongcheng Districts. In contrast, the population flow was mainly between Xinfadi Wholesale Market and the southern areas, especially Fengtai and Daxing Districts (figure 2A). Traffic volume between the market and subdistricts is shown in figure $2 \mathrm{~B}$, which indicates that cases are mainly distributed in subdistrict with higher traffic volume from the market, especially in the southern areas. Huaxiang Town and Xincun Residential District, Xinhongmen Town and Huangcun Town had the highest traffic volume with the top three number of cases. Generally speaking, the shorter the commuting distance $(p=0.019)$ and the larger visitor flow volume $(\mathrm{p}=0.021)$ of an area to Xinfadi
Wholesale Market, the higher risk of people being infected (figure 2C). However, Huangcun Town and Yongdinglu Residential District, farther from Xinfadi Wholesale Market, also had a large number of cases, which was mainly due to the clusters of PepsiCo's factory and East Yuquan Market, respectively. The findings suggest that SARS-CoV-2 was mainly transmitted through person-toperson, rather than through contaminated products.

Taking COVID-19 containment as the most pressing priority, various public health measures had been implemented in Beijing (online supplemental figure S1). The effective reproduction number $R_{t}$ was up to 6.6 with a wide CI at the very beginning of the outbreak, when the virus silently occurred and transmitted in Xinfadi Wholesale Market (figure 3A). Then, $R_{t}$ dramatically dropped to the level of 3.0 on 13 June, when Xinfadi Wholesale Market was suspended and the surrounding communities were closed and disinfected. After that, the relative traffic volume in all subdistricts dropped significantly by $20 \%$ (figure 3B), and the decrement rate of high-risk, median-risk and low-risk areas were $50 \%, 35 \%$ and $20 \%$, respectively. Compared with $>80 \%$ traffic volume reduction in the first wave of COVID-19 outbreak in China, this Xinfadi outbreak has less impact on urban traffic. ${ }^{17}$ Since the first case was reported on 11 June, blanket screening has been conducted to find out people who had been to Xinfadi Wholesale Market. As of 2 July, a total of 8500 close contacts have been traced and quarantined (figure 3C). From 14 June onwards, the authorities tested all purchasers, people working at Xinfadi Wholesale Market and residents living in the high-risk and medium-risk area. The intervention action is called 'Testing Everyone Who Should be Tested'. During this week, the proportion of positive tests was relatively high, although the daily amount of NAT was quite small. Since 20 June, any individuals who are willing to have a NAT can go to the medical institutions. This policy is called 'Testing Everyone Who Wants to be Tested'. In this phase, the amount of NAT in Beijing increased significantly; however, the positive number decreased to a low level. As of 5 July 2020, over 10 million people have been tested (figure 3D). On 16 June, Beijing authorities raised their public health emergency response from the level 3 to level 2. $R_{t}$ maintained downward trend and finally decreased to below 1 on 18 June, 2 weeks after the first case occurred (figure 3A). Cases of COVID-19 were basically identified through the three approaches. First, active medical treatment (patients go to medical institutions for diagnosis actively), which includes 93 cases $(27.8 \%)$. Second, closecontact tracing, which includes 92 cases $(27.5 \%)$. The third is by community-based NAT, including 150 cases and occupying $44.8 \%$. The proportion of cases screened by close-contact tracing and NAT increased during the outbreak (figure 3E).

Figure 4 shows the results of model simulations. We first fitted the modified SEIR model (online supplemental figure S6) to daily incidence from 4 June 2020 to 5 July 2020 , and simulated the spread of epidemic under several 

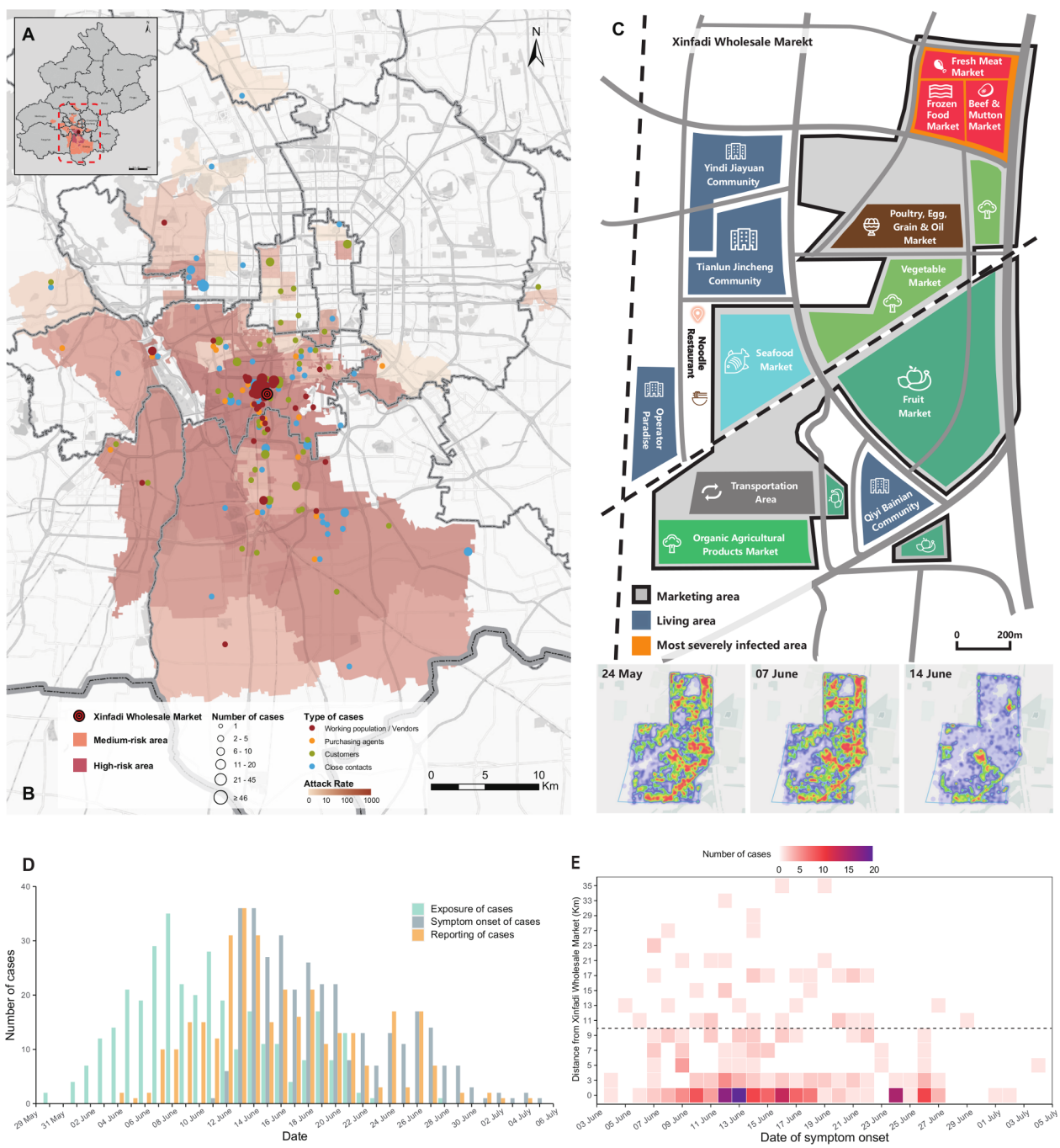

Figure 1 Spatiotemporal characteristics of COVID-19 outbreak linked to Xinfadi Wholesale Market in Beijing in June 2020. (A) The distribution of medium-risk and high-risk areas during the outbreak. (B) The subdistrict-level attack rate of COVID-19 in Beijing. Communities or subdistricts with confirmed cases are marked on the map based on the number and type of cases. (C) The layout and daily visitor flow volume of Xinfadi Wholesale Market. (D) Epidemic curve (exposure, symptom onset and reporting date) of cases. (E) Shows the matrix between the number of cases and the date of symptom onset and distance to the market.

scenarios with different combinations of NPIs. Figure 4A provides a general description of the results, which plots the number of cumulative cases under all scenarios by 5 July, 1 month after the first case of this outbreak developed symptoms. Under the scenario that all NPIs we investigate (localised lockdown, close-contact tracing and community-based NAT) are absent, there would be a total of 25708 COVID-19 cases (95\% CI 13657 to 40625 ) in Beijing, which is an 76.7-fold increase compared with the actual scenario (online supplemental figure S7A). Figure 4B plots the individual impacts of the NPIs. For the scenario with only localised lockdown, close-contact tracing or community-based NAT is conducted, there would be 2175 (95\% CI 1368 to 2990), 2651 (95\% CI 2006 to 3417 ) or 1291 (95\% CI 1039 to 1602) cases as of 5 July, which are 6.5-fold, 7.9-fold or 3.9-fold increase, respectively. Among them, the scenario with only communitybased NAT produces the least cumulative cases. We also simulated the effects of paired NPIs, that is, only one of the NPIs lacks. For the scenario without localised lockdown intervention, without close-contact tracing or without community-based NAT, the total COVID-19 cases in Beijing by 5 July could be up to 472 (95\% CI 410 to 564), 573 (95\% CI 477 to 682 ) or 1107 (95\% CI 828 to 1387), which are 1.4-fold, 1.7-fold or 3.3-fold increase compared with the actual scenario, respectively (online supplemental figure S7B). In order to evaluate the effectiveness of the targeted test policy, we estimated a scenario without NAT after 20 June, which would be a total of 398 (95\% CI 352 to 450 ) cases by 5 July, with only 63 more cases than the actual scenario. Therefore, the targeted test policy in the early stage of the outbreak has a significant effect on containing infections. Overall, among all NPIs we investigated, community-based NAT was the most effective intervention under single NPI scenarios. In contrast, close-contact tracing, combined 

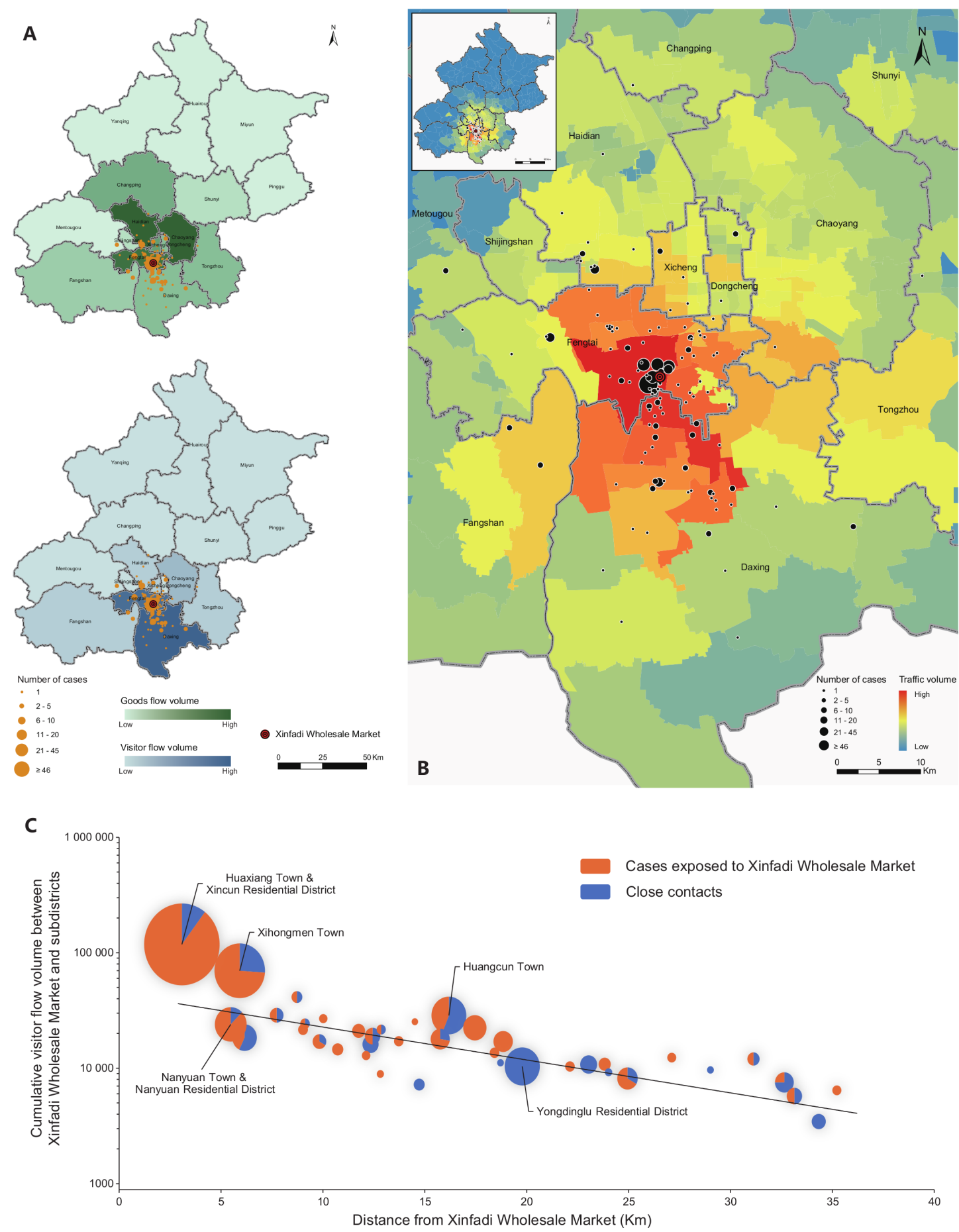

Figure 2 The correlation of human mobility and goods transportation from Xinfadi Wholesale Market with the COVID-19 outbreak in Beijing. (A) Spatial distribution and flow volume of goods and visitors from Xinfadi Wholesale Market. (B) Traffic volume of all subdistricts from the market based on data of an online ride-hailing platform. (C) The distance-visitor flow volume scatter plot, and pie plots represent proportions of different exposure cases.

with community-based NAT performed the best among paired NPIs scenarios. Moreover, the effects of combined interventions were more significant compared with single or partial interventions (online supplemental table S5 for the main analysis and online supplemental figure S6-S11 for sensitivity analyses). Performance of models in the main and sensitivity analyses are summarised in online supplemental table S12, figure S8A and S8B. Trace plots were used for judging the convergence of model fitting (online supplemental figure S9).

\section{DISCUSSION}

The resurgent COVID-19 outbreak in Beijing highlights a new challenge for the pandemic control. The origin of this outbreak is still unknown. One possible origin was 


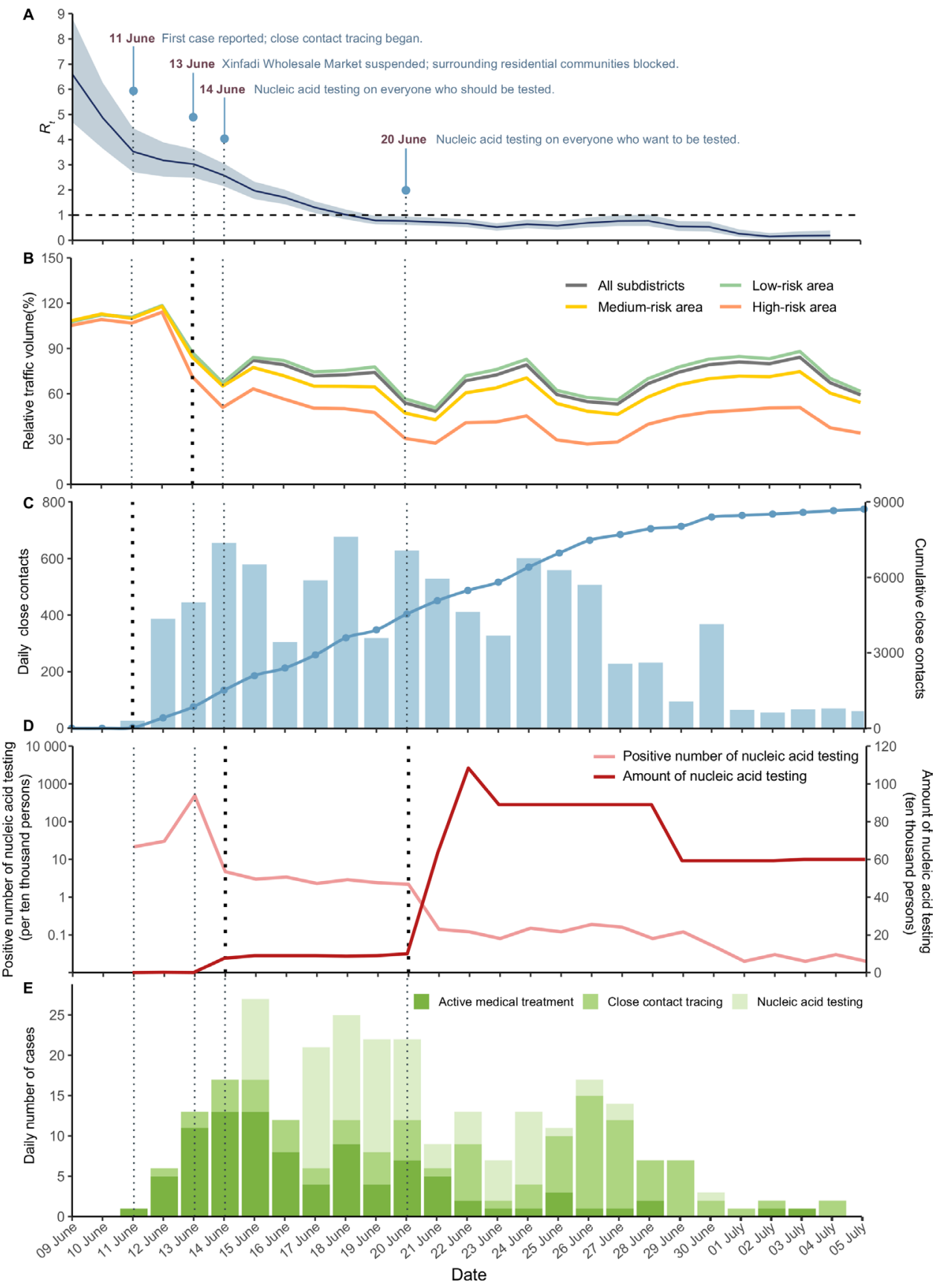

Figure 3 The efficacy of containment measures implemented in Beijing. (A) Average $R_{t}$ and $95 \% \mathrm{Cl}$. The critical value of $R_{t}=1$ is marked with a horizontal dash line. The moment of important events and public health control measures are marked by date. Each dot line in bold corresponds to the observation that this measure affect. (B)-(D) Observations (relative traffic volume, close contacts and nucleic acid testing) change during the outbreak. (E) The daily number of cases confirmed by three approaches, that is, active medical treatment, close contact tracing and nucleic acid testing. $R_{t}=$ effective reproduction number.

the contaminated frozen products, in which the longer stability of the virus could be expected under cold condition. ${ }^{18-22}$ Another possible origin was the presymptomatic or asymptomatic people working in the market infected with the virus. No matter its origin, Xinfadi Wholesale Market has been a potential 'virus amplifier' where uncontainable spread inside and outside the market facilitated. Xinfadi Wholesale Market is the largest wholesale market in Asia, occupying 1680 acres with the highest sales volume. ${ }^{23}$ The market has the trade volume as high as 1749 million tons in 2019, and supplied around $90 \%$ of total supplies of fruit and vegetable, and $15 \%-20 \%$ of beef and mutton in Beijing. The transport volume of the market is around 60000 people per day and 30000 vehicles per day. The market was roughly divided into nine areas based on commodity category. The COVID-19 infections were detected from all areas and the areas for frozen seafood and beef and mutton were most severely infected both in cases and environmental samples. ${ }^{24}$ The areas for storage, processing and sale of frozen goods or products 
A

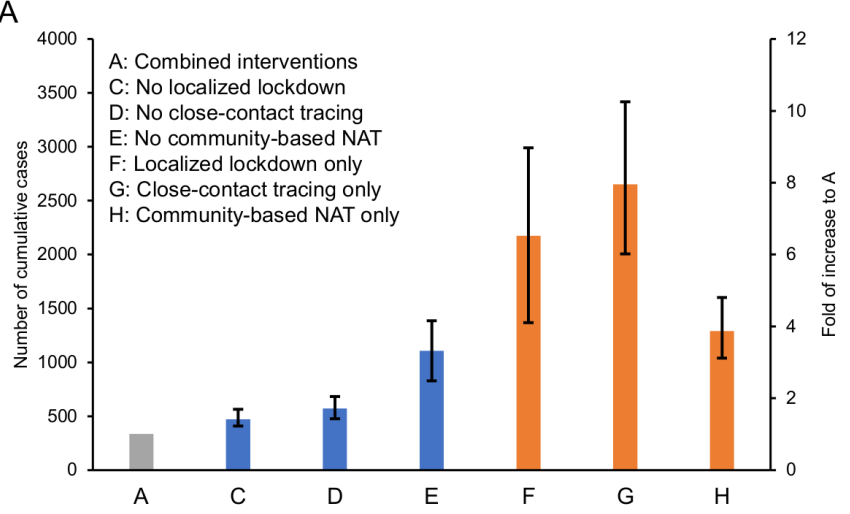

B

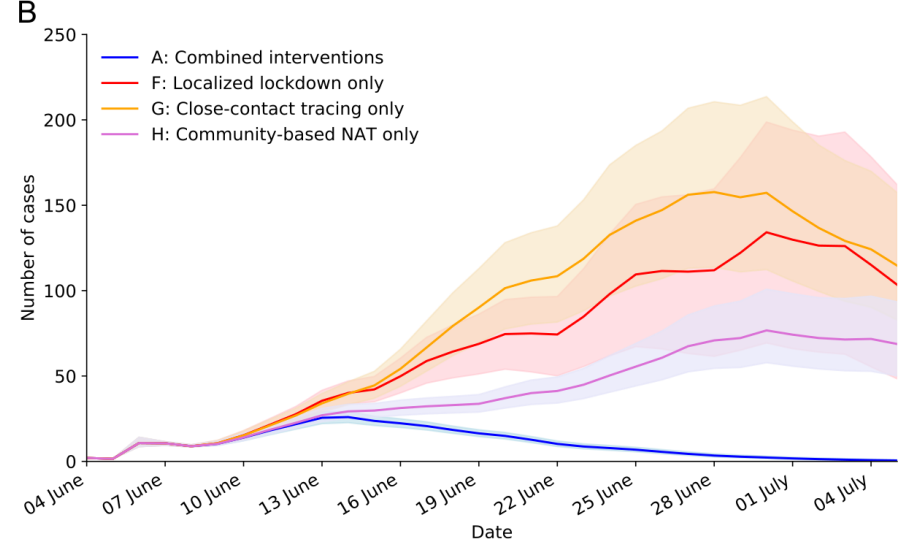

Figure 4 The results of simulations under different combinations of NPIs. (A) Cumulative cases by 5 July under scenario from $(A)-(H)$ and increase of fold for scenario $(C)-(H)$ compared with (A). We exclude the plotting of scenario $(B)$ in $(A)$ because of its large value. (B) The solid colour line segment represents the median of replicated experiments, and the shadow represents $95 \%$ $\mathrm{Cl}$ of the replicates. During the experiments, we ran the Metropolis-Hastings Markov chain Monte Carlo simulation for 10 million iterations and sampled at every 1000th step. NAT, nucleic acid testing; NPIs, non-pharmaceutical interventions.

are at a high risk of maintaining and disseminating the virus. During the ongoing global pandemic, great attention and control measures focusing on big wholesale markets should be taken.

In this study, we suggested that exposed population rather than contaminated sales was the principal reason for the spread of the virus in Beijing. People working in Xinfadi Wholesale Market who had constant exposures to the contaminated environment have the highest infection rate. It may also explain the relatively higher proportion of male and younger median age of Beijing cases than the overall country cases. ${ }^{25}$ Occupational health guideline or legislation on COVID-19 prevention and control for employers in wholesale market, or wet market should be strengthened. We identified that the exposed human not contaminated goods have mainly transmitted the virus. The special humidity and temperature or even particular processing activities occurred in the market might favour the virus containment, however, the infectivity of the virus contained in the products seemed to be dramatically decreased after a long trip of transportation. Our observation that most cases distributed around 10 $\mathrm{km}$ distance to Xinfadi Wholesale Market also supported this hypothesis.

Beijing authorities downgraded the emergency response to COVID-19 to level 3 on 6 June, and masks were no longer mandatory. Facing the hard-won adjustment of the response level and unbearable heat in early June, some citizens in Beijing did not wear masks when they were outdoors. Xinfadi Wholesale Market is a place with high people mobility and density, where people have more chances to contact others closely, which has facilitated the quick transmission from person to person.

Learning from the experience and lessons from the first epidemic wave in Wuhan, rapid and targeted control measures were implemented in Beijing (online supplemental figure S1). Almost at the same time as the epidemic started, Beijing authorities have responded quickly to shut down Xinfadi Wholesale Market (on 13
June) and enhanced its emergency response to level 2 (on 16 June) again. During the outbreak, authorities only locked down streets and communities in high-risk areas, rather than confining everyone in Beijing to their homes. All people working or living in these areas, or having exposed to Xinfadi Wholesale Market were required to take nucleic acid tests and self-quarantine for further observation. Considering thousands of people might be exposed to the market, the authorities used big data and artificial intelligence technologies to track individuals' movements and identify those who had visited Xinfadi Wholesale Market. ${ }^{26}$ By those combined NPIs, Beijing epidemic was promptly controlled and only self-sustained for around 2 weeks.

Because most of cases were mild and easy to be misdiagnosed and under-reported, we evaluated a scenario in which the disease was unnoticed and no intervention was conducted. From 4 June to 5 July, there would be around 25000 COVID-19 cases in Beijing without any intervention. Before the travel restrictions and strict interventions implemented on 23 January in Wuhan city, a total of 830 COVID-19 cases was documented, ${ }^{27}$ although some mathematical model suggested a substantial undocumented infection before the lockdown of Wuhan city. ${ }^{28}$ To some extent, our model indicated that the precise identification of Xinfadi Wholesale Market as the epicentre and the decisive conduction of public health interventions in Beijing has avoided a similar severe epidemic as in Wuhan. Our improved model could be used to evaluate effects of the different strategies on active case finding and management, compared with previous modelling on COVID-19 control. ${ }^{29-31}$ We showed that NAT is the most important way to identify the cases; furthermore, the NAT targeted on Xinfadi-exposed population contributed to around $91.8 \%$ effect of NAT on the disease control. The city has at first tested nearly all the people who have exposure history to Xinfadi Wholesale Market and related cases, then the testing was enlarged to the residents living in the high-risk and medium-risk areas and employees in 
key industries, such as catering, supermarkets, express delivery, social welfare institutions, medical and health institutions, bank counter staff, transportation and beauty salons. As to combat against COVID-19, the increase in NAT capability by adopting high-technique equipment and high-throughput technology is essential in the future.

Our research provides a constructive framework to analyse the transmission dynamics of the emergent cluster of COVID-19 from two perspectives, including (i) detailed spatiotemporal analysis based on individual recordings on laboratory-confirmed COVID-19 case data and mobility data of both visitors and products; (ii) modified epidemiological modelling with time-variant parameters inferred from data for in-depth analysis on the effects of NPIs. However, we do acknowledge several limitations. In our simulations, we assumed that the medical resources were unlimited and the efficiency of all the NPIs would not decrease even when the infected population grows in a short period. In reality, as the epidemic develops unnoticeably, medical resources (eg, testing reagents) would become strained and even shortage, and the difficulty of conducting epidemiological investigations would increase as well, which may reduce the efficiency of NPIs. Besides, some of the NPIs implemented in Beijing, like wearing masks or disinfection of public places, were not considered in this study. Finally, our approach for model calibration may not be applicable to all countries or regions. Specifically, we only used the reported infections to calibrate our model, which may lead to errors for areas with the issue of under-reporting. However, due to the large-scale testing in China, this problem can be avoided in our study.

\section{CONCLUSION}

Although the origin of Beijing outbreak is still a mystery and requires further tracing, we highlight that big marketrelated outbreak is a significant event and may lead to severe epidemic without proper control measures. The key to tackling is to target the market-exposed people. Enlarged NAT and big data contact tracing are efficient approaches to be implemented.

\section{Author affiliations}

${ }^{1}$ State Key Laboratory of Pathogen and Biosecurity, Beijing Institute of Microbiology and Epidemiology, Academy of Military Medical Sciences, Beijing, China

${ }^{2}$ Institute of EcoHealth, School of Public Health, Cheeloo College of Medicine, Shandong University, Jinan, Shandong, China

${ }^{3}$ School of Computer Science and Engineering, Beihang University, Beijing, China

${ }^{4}$ Peng Cheng Laboratory, Shenzhen, China

${ }^{5} \mathrm{MOE}$ Engineering Research Center of ACAT, School of Computer Science and Engineering, Beihang University, Beijing, China

Contributors WC, NJ, JW and XC designed research. WC, NJ, JW, XC, LZ, YZ, XL, RY, KM, J-FJ, BJ, ZX, H-HS, QL and XW collected and analysed data. WC, NJ, JW, XC, $L Z, Y Z, X L, R Y$ and $K M$ prepared the manuscript. The final version of the manuscript was contributed to and approved by all authors. The corresponding author has full access to all the data in the study.

Funding This work was supported by the National Natural Science Foundation of China (81621005, 82161148011, 92046010 and 72171013), the State Key
Research Development Program of China (2020YFC0846300, 2019YFC1200202, 2019YFC1200401 and 2019YFC1200505), the China Postdoctoral Science Foundation (2020T130069ZX).

Competing interests None declared.

Patient consent for publication Not required.

Ethics approval As this study constituted public health surveillance rather than research in human beings, ethical approval from institutional review boards was not required. All information on individual persons had been anonymised.

Provenance and peer review Not commissioned; externally peer reviewed.

Data availability statement № data are available. The data set used to support the findings of this study are not publicly available as proprietary or confidential information was included.

Supplemental material This content has been supplied by the author(s). It has not been vetted by BMJ Publishing Group Limited (BMJ) and may not have been peer-reviewed. Any opinions or recommendations discussed are solely those of the author(s) and are not endorsed by BMJ. BMJ disclaims all liability and responsibility arising from any reliance placed on the content. Where the content includes any translated material, BMJ does not warrant the accuracy and reliability of the translations (including but not limited to local regulations, clinical guidelines, terminology, drug names and drug dosages), and is not responsible for any error and/or omissions arising from translation and adaptation or otherwise.

Open access This is an open access article distributed in accordance with the Creative Commons Attribution Non Commercial (CC BY-NC 4.0) license, which permits others to distribute, remix, adapt, build upon this work non-commercially, and license their derivative works on different terms, provided the original work is properly cited, appropriate credit is given, any changes made indicated, and the use is non-commercial. See: http://creativecommons.org/licenses/by-nc/4.0/.

\section{ORCID iDs}

Lin Zhao http://orcid.org/0000-0003-0545-4194

Yuhao Zhou http://orcid.org/0000-0002-0237-5813

\section{REFERENCES}

1 World Health Organization. Weekly epidemiological update on COVID-19-13, 2021. Available: https://www.who.int/publications/ m/item/weekly-epidemiological-update-on-covid-19-13-april-2021 [Accessed 15 Apr 2021]

2 Zhu N, Zhang D, Wang W, et al. A novel coronavirus from patients with pneumonia in China, 2019. N Engl J Med Overseas Ed 2020;382:727-33.

$3 \mathrm{Li} \mathrm{Q}$, Guan X, Wu P, et al. Early transmission dynamics in Wuhan, China, of novel Coronavirus-Infected pneumonia. N Engl J Med 2020;382:1199-207.

4 Zhang $X$, Tan Y, Ling Y, et al. Viral and host factors related to the clinical outcome of COVID-19. Nature 2020;583:437-40.

5 Beijing Center for Diseases Prevention and Control. Epidemic situation, 2020. Available: https://www.bjcdc.org/ColumnAction.do? dispatch $=$ getEjPage\&id=4473\&clD=4493 [Accessed 05 Jul 2020].

6 Watts A. Coronavirus cluster in Beijing is a "significant event", WHO says, 2020. Available: https://edition.cnn.com/world/live-news/ coronavirus-pandemic-06-16-20-int//h_654790a09880fff1931d13ac 5d531dcd [Accessed 08 July, 2020].

7 Chinese S. Molecular evolution of the SARS coronavirus during the course of the SARS epidemic in China. Science 2004;303:1666-9.

8 Wang W, Artois J, Wang X, et al. Effectiveness of Live Poultry Market Interventions on Human Infection with Avian Influenza A(H7N9) Virus, China. Emerg Infect Dis 2020;26:891-901.

9 Liu J. Research on logistics efficiency of xinfadi agricultural product wholesale- - taking vegetable wholesale for example master's thesis. Beijing Jiaotong University, 2015.

10 Cori A, Ferguson NM, Fraser C, et al. A new framework and software to estimate time-varying reproduction numbers during epidemics. Am J Epidemiol 2013;178:1505-12.

11 de Vlas SJ, Feng D, Cooper BS, et al. The impact of public health control measures during the SARS epidemic in mainland China. Trop Med Int Health 2009;14 Suppl 1:101-4

12 Haario H, Saksman E, Tamminen J. An adaptive Metropolis algorithm. Bernoulli 2001;7:223.

13 Brooks SP, Gelman A. General methods for monitoring convergence of iterative simulations. J Comput Graph Stat 1997;7:434-55.

14 Patil A, Huard D, Fonnesbeck C. PyMC : bayesian stochastic modelling in. J Stat Softw 2010;35:81. 
15 He X, Lau EHY, Wu P, et al. Temporal dynamics in viral shedding and transmissibility of COVID-19. Nat Med 2020;26:672-5.

16 Hao X, Cheng S, Wu D, et al. Reconstruction of the full transmission dynamics of COVID-19 in Wuhan. Nature 2020:584:420-4.

17 Migration B. Databases of Intra-city travel intensity, 2020. Available: https://qianxi.baidu.com [Accessed 05 Jul 2020].

18 Matson MJ, Yinda CK, Seifert SN. Effect of environmental conditions on SARS-CoV-2 stability in human nasal mucus and sputum. Emerg Infect Dis 2020;26:2276-8.

19 Kratzel A, Steiner S, Todt D, et al. Temperature-Dependent surface stability of SARS-CoV-2. J Infect 2020;81:452-82.

20 Cimolai N. Environmental and decontamination issues for human coronaviruses and their potential surrogates. J Med Virol 2020;92:2498-510.

21 Casanova L, Rutala WA, Weber DJ, et al. Survival of surrogate coronaviruses in water. Water Res 2009;43:1893-8.

22 Wang J, Tang K, Feng K, et al. Impact of temperature and relative humidity on the transmission of COVID-19: a modelling study in China and the United States. BMJ Open 2021;11:e043863.

23 Zhao L. Beijing's new COVID-19 cluster: Inside Xinfadi biggest wholesale market in Asia, 2020. Available: https://news.cgtn.com/ news/2020-06-14/COVID-19-in-Beijing-Inside-Xinfadi-biggestwholesale-market-in-Asia-RjgKHh6HPW/index.html [Accessed $08 \mathrm{Jul}$ 2020].
24 The People's Government of Beijing Municipality. COVID-19 situation report, 2020. Available: http://www.beijing.gov.cn/shipin/ xxgzbdfyyqfb/ [Accessed 08 Jul 2020].

25 Qian J, Zhao L, Ye R-Z, et al. Age-Dependent gender differences in COVID-19 in mainland China: comparative study. Clin Infect Dis 2020;71:2488-2494.

26 Lin L, Hou Z. Combat COVID-19 with artificial intelligence and big data. J Travel Med 2020;27:taaa080.

27 National Health Commission. Update information on COVID-19 in China, 2020. Available: http://wjw.wuhan.gov.cn/ztzl_28/fk/yqtb/ 202004/t20200430 1196683.shtml [Accessed 24 Jan 2020].

28 Li R, Pei S, Chen B, et al. Substantial undocumented infection facilitates the rapid dissemination of novel coronavirus (SARSCoV-2). Science 2020;368:489-93.

29 Lai S, Ruktanonchai NW, Zhou L, et al. Effect of nonpharmaceutical interventions to contain COVID-19 in China. Nature 2020;585:410-3.

30 Chinazzi M, Davis JT, Ajelli M, et al. The effect of travel restrictions on the spread of the 2019 novel coronavirus (COVID-19) outbreak. Science 2020;368:395-400.

31 Zhang J, Litvinova M, Liang Y, et al. Changes in contact patterns shape the dynamics of the COVID-19 outbreak in China. Science 2020;368:1481-6. 